\title{
Éditorial:
}

\section{Bâtir la gérontologie d'ici: un tremplin pour la reconnaissance internationale?}

Un champ d'activités scientifiques s'inscrit dans le tissu social par des institutions: des unités de formation et de recherche universitaires, des associations scientifiques et éducatives, des revues scientifiques, des organismes de subvention de la recherche. La cristalisation de ces institutions est le résultat d'un jeu complexe d'acteurs engagés et clairvoyants; ils savent accéder à des ressources, les mobiliser pour, à la fois, assurer la continuité de leur projet de construction du champ, produire de la connaissance et former des praticiens. Jusqu'ici, la gérontologie au Canada a profité de la présence et de l'engagement de visionnaires et de bénévoles dévoués qui ont mis sur pied, au cours du dernier quart de siècle, des institutions scientifiques et professionnelles tout en déployant une activité de recherche et de formation exceptionnelle à plusieurs égards. Au Québec, le réveil fut plus tardif, moins articulé autour de centres ou de groupes de recherche. La gérontologie au Canada et au Québec peut-elle continuer sur cette lancée? Et quelles sont les perspectives d'avenir?

La gérontologie canadienne s'est développée dans une période relativement favorable à son expansion. Dans les années '70 et à la fin des années ' 80 , se préparaient certes les discours néo-libéraux courants aujourd'hui, mais les grands programmes sociaux s'implantaient, se rodaient et se consolidaient. Le système d'assurance-maladie universelle, en particulier, semblait prendre une expansion intéressante, mais modeste, du côté des soins de longue durée par l'adoption de nouveaux modes de paiement de transfert du fédéral vers les provinces, par l'introduction progressive de soins à domicile, par un déplacement du rôle de l'hébergement en institution des personnes âgées. Enfin, malgré tous les discours sur la marginalisation des personnes âgées, les tentatives de désindexation des pensions de vieillesse fédérales se butaient à la résistance des organisations de personnes âgées et à la solidarité des autres groupes d'âge.

Dans ce contexte social, naît l'Association canadienne de gérontologie; le Conseil de recherche en sciences humaines met sur pied son programme de financement des infrastructures de recherche universitaire en gérontologie; La Revue canadienne du vieillissement est fondée. Le gouvernement fédéral établit divers programmes, directions et groupes consultatifs sur les personnes âgées. Pêle-mêle et sans souci d'exhaustivité ni d'exactitude chronologique, citons le Conseil national du troisième âge, le programme «Nouveaux Horizons,» le programme de recherche sur l'autonomie des aînés. Santé Canada finance des recherches sur l'autonomie des aînés, sur la maladie d'Alzheimer, et Statistique Canada s'engage dans des recherches sur les problèmes fonctionnels des personnes âgées. Certaines des enquêtes so- 
ciales générales se consacrent en partie à l'étude d'aspects du vieillissement: les réseaux et le soutien social par exemple. D'autres projets d'envergure voient le jour et obtiennent des fonds, démontrant le caractère dynamique de la gérontologie d'ici. Pour ne donner qu'un exemple, le seul projet de sciences sociales financé dans le cadre de la première vague des centres d'excellence est CARNET: Le réseau canadien de recherche sur le vieillissement, un programme de recherche d'une équipe de chercheurs en gérontologie sociale.

Et puis, on le dit, l'époque a changé. Par exemple, la sécurité de la vieillesse n'est plus un programme fédéral universel. Le temps ne serait plus à l'implantation de grands programmes sociaux. La rhétorique politique est dominée par la dialectique du déficit public et de la réduction du rôle de l'État. Au Canada, il n'y a probablement qu'un seul programme social aux principes intouchables: le régime d'assurance-maladie universelle. Le Québec l'a même étendu aux médicaments avec un programme mixte privé et public qui garantit à tous les citoyens du Québec une couverture minimale. Mais, l'introduction de ce programme origine autant de la nécessité de permettre l'accès à des médicaments coûteux, autrefois distribués gratuitement au cours de l'hospitalisation, qu'au soucis du gouvernement québécois de récupérer annuellement 200 millions de dollars du régime d'assurance-médicaments précédent qui ne couvrait que les personnes âgées et les assistés sociaux. Paradoxalement, c'est pour réduire le coût, pour l'État, du programme qu'on l'a universalisé!

Tandis que le climat socio-politico-économique se transformait, la structure de recherche en gérontologie établie au cours des années quatre-vingt s'étiolait. Dans le monde francophone, le seul centre de recherche universitaire, celui de l'Université de Moncton, a failli disparaître; ses activités sont actuellement réduites. Au Canada anglais, des centres de recherche en gérontologie ont fermé, d'autres se sont transformés, et la survie et l'activité des centres les plus productifs semblent être liées à des personnes combatives et dynamiques plutôt qu'à l'établissement d'une forte tradition de recherche qui repose sur la pérennité d'efforts continus.

Il ne s'agit pas d'être alarmistes. Les chercheurs en gérontologie, pour être productifs, n'ont pas nécessairement besoin de centres de recherche dédiés. Mon expérience de rédacteur-en-chef de La Revue canadienne du vieillissement (RCV) m'a montré que, de 1990 à 1996, le nombre de manuscrits soumis et publiés a au moins doublé alors que des temps plus difficiles étaient annoncés et nous avaient même rattrapés. Toutefois, durant la dernière année de mon mandat, le nombre de manuscrits soumis a soudainement chuté. Est-ce l'amorce d'une diminution durable ou un incident de parcours? Et s'il s'agit des premiers moments d'un mouvement à la baisse, celui-ci est-il limité à la $R C V$ ? Ou réflète-t-il une diminution de la production scientifique en gérontologie? Peut-être dans toutes les revues canadiennes de sciences sociales?

Il n'est pas indifférent de savoir ce qui s'est passé. Si la baisse du nombre de manuscrit soumis à la $R C V$ ne s'applique qu'à la $R C V$, cela signifie la 
désaffectation relative des chercheurs d'ici pour une revue scientifique canadienne. La fidélité assez remarquable des chercheurs en gérontologie pour la $R C V$ s'effilocherait au bénéfice de revues étrangères. Autrement dit, les chercheurs en gérontologie se comporteraient désormais comme leurs collègues d'autres disciplines. La $R C V$, comme beaucoup de revues scientifiques canadiennes, serait condamnée à jouer un rôle local, même si, au cours des premières années de son histoire, elle promettait de percer sur le marché international.

Une diminution de la production scientifique en gérontologie serait encore plus inquiétante. Il faudrait voir si d'autres disciplines évoluent de manière semblable. Les budgets raréfiés des organismes publics de subvention de la recherche et l'encouragement aux liens entre recherche universitaire et secteur privé ou établissements publics sont deux des politiques gouvernementales actuelles qui pourraient être responsables d'une baisse de la production d'informations scientifiques publiques. ${ }^{1}$

Un autre facteur est inquiétant pour l'avenir de la gérontologie. Non seulement les fonds des grands organismes fédéraux de financement de la recherche - CRSH, CRM, PNRDS - sont-ils moins bien pourvus qu'auparavant, mais des organismes qui ont joué un rôle vital dans le développement de la recherche sur les personnes âgées, comme le PNRDS, se réorientent vers la recherche non plus appliquée, mais dirigée. La gérontologie a toujours eu des soucis pratiques; elle n'a pas péché par excès de théorisation. Aussi est-il faux de prétendre que les chercheurs en gérontologie ont négligé les problèmes pratiques du vieillissement. Seulement, ils définissaient les aires de recherche qui leur semblaient les plus intéressants et prometteurs. C'est ainsi que la recherche canadienne s'est faite remarquée sur la scène internationale par les travaux sur l'utilisation des services de santé par les personnes âgées des groupes de l'Université de ColombieBritannique et de l'Université du Manitoba, sur le soutien social et la famille par des chercheurs de l'Université de Victoria et de l'Université de Guelph, par des recherches sur les travailleurs âgés à l'Université de Toronto. D'autres chercheurs du Québec, du Nouveau-Brunswick, de Terre-Neuve et de l'Alberta se sont aussi distingués par leurs travaux en psychologie et sociologie du vieillissement. La liste pourrait s'allonger et je demande pardon immédiatement à tous ceux que je n'ai pas nommés. Malgré cette remarquable production scientifique appliquée, aujourd'hui des organismes de subvention ont la prétention de diriger les recherches vers des thèmes «porteurs». Ce mouvement vers le financement sélectif des sujets de recherche, choisis par des décideurs politico-bureaucratiques et accompagnés de consultations préalables du milieu, n'a pas pris en considération l'histoire récente de la recherche d'ici en gérontologie, son implantation profonde dans la recherche appliquée et l'engagement des chercheurs envers les aspects les plus problématiques du vieillissement.

Cette réorientation récente de certains des organismes de financement de la recherche vers la recherche dirigée s'est rarement accompagnée de ressources adéquates; elle se traduit plutôt par une restriction des crédits 
alloués à la recherche. L'expérience du «Programme de recherche sur l'autonomie des aînés" (PRAA) pourrait contredire cette assertion. Mais la probabilité qu'une expérience semblable se répète est très faible, sinon nulle, et la durée de vie des projets du PRAA n'est que de trois ans, limitant les chercheurs aux devis de recherche transversaux ou à l'analyse de données secondaires.

Voici l'autre des grands problèmes de la recherche en gérontologie: les modalités de financement de la recherche rendent extrêmement difficile l'exécution de projets innovateurs, d'envergure, risqués et hors des sentiers connus. Ainsi, le risque est grand de marginaliser la recherche gérontologique canadienne. Pernons un exemple. Linda George (1996), dans un chapitre de la quatrième édition de Handbook of Aging and the Social Sciences, prétendait que, sauf exception, il y avait peu à espérer des devis transversaux dans les recherches sur le soutien social aux personnes âgées; il faudrait donc s'engager dans des recherches longitudinales. Au-delà de ce qu'il y a d'exagéré dans cet énoncé, il comporte une grande leçon: dans la mesure où les anathèmes et les abjurations des grandes figures de la recherche finissent par être partagés par les moindres figures et que les uns et les autres les appliquent à l'arbitrage des articles de revues, ces normes gouvernent ce qui est publiable ou non.

En clair, si l'opinion de madame George se répand, il deviendra de plus en plus difficile de publier des travaux de recherche sur le soutien social qui ne seront pas longitudinaux. Et il est vrai que les études longitudinales permettent d'obtenir des réponses plus satisfaisantes à certaines questions de recherche que les devis transversaux. Or, le financement, l'organisation et l'exécution de la recherche longitudinale ne sauraient suivre les mêmes règles que celles des projets ad hoc. Et les chercheurs du Canada et du Québec ont été formés à penser, rédiger et exécuter des projets de courte durée financés sur des périodes de deux à trois ans. Ils n'ont fait que se conformer aux cycles de financement imposés par les organismes de financement de la recherche.

La recherche gérontologique dans la communauté canadienne peut progresser modestement mais sûrement, avec les ressources disponibles à tous les chercheurs de toutes les disciplines. Il y a, et il y aura probablement toujours, des chercheurs intelligents et entreprenants qui formeront autour d'eux de petites équipes dont les publications seront destinées en priorité aux revues scientifiques américaines et internationales. Ces personnes pourront aussi jouer le rôle de consultant auprès d'organismes fédéraux, provinciaux, régionaux ou locaux.

Il y a une autre façon de voir. La recherche gérontologique peut fonder son action sur des institutions d'ici pour contribuer à la fois au développement de la gérontologie internationale et à la définition et la résolution des enjeux du vieillissement dans nos sociétés. Ce qui implique une contribution active de l'Association canadienne de gérontologie (ACG) aux débats sur le vieillissement fondée sur les résultats de la recherche, une présence des meilleurs chercheurs d'ici et d'ailleurs aux congrès annuels de l'ACG et 
l'utilisation et la promotion de La Revue canadienne du vieillissement comme média normal de communication des résultats de recherche. Cela implique aussi une perspective nouvelle sur l'organisation de la recherche où l'innovation, l'ambition et le risque sont récompensés.

Il y a plusieurs façons d'inciter à l'innovation, de promouvoir la qualité et la con tinuité des efforts de recherche. Il n'est pas de mon propos d'aborder ce sujet ici. Il est cependant important que des moyens adéquats soient utilisés, et des moyens adéquats ne sont mis qu'à la disposition d'objectifs valorisés et prioritaires. Or, j'ai le sentiment, et je souhaite me tromper, que le vieillissement n'est plus, pour les gouvernements et même pour les intellectuels du domaine de la santé, une priorité. En effet, le rapport du Forum national sur la santé (1997) fixe comme priorité un groupe d'âge: l'enfance. Ce choix se défend et se comprend bien. Le seul domaine prioritaire du Forum qui réfêre au vieillissement est celui des services à domicile. Une priorité étrange, limitée, puisqu'elle n'est pas vraiment située dans une réflexion plus vaste sur l'organisation de l'ensemble des soins et services aux personnes âgées. Qui plus est, on peut sentir un changement dans l'orientation de la société envers les personnes âgées. Si elles furent le fait de la sollicitude de la société, une sollicitude mâtée de paternalisme, elles tombent aujourd'hui dans une indifférence relative. Toute relative, il est bien vrai, puisque même si les priorités des politiques de santé et de bien-être se dirigent dorénavant vers l'enfance et l'adolescence, les services et le soutien financier public aux personnes âgées resteront des préoccupations «administratives». La recherche sèra sollicitée pour trouver des solutions aux problèmes concrets d'organisation des programmes publics destinés aux personnes âgées; mais cette recherche restera sans perspectives, à moins qu'elle réussisse à poser elle-même les bonnes questions et à négocier, par sa qualité et sa force, les ressources nécessaires pour le développement de ses propres programmes de recherche.

\section{Note}

1 Une dernière explication de la diminution du nombre de manuscrits soumis est plus prosaïque: le plus grand nombre d'articles à publier a allongé les délais de publication et les chercheurs ont évité la $R C V$. S’il peut y avoir un peu de vérité dans cette explication, elle ne m'apparait toutefois pas satisfaisante.

\section{Références}

Forum national sur la santé. (1997). La santé au Canada: un héritage à faire fructifier. Rapport final du Forum national sur la santé. Ottawa: Ministère des Travaux publics et Services gouvernementaux.

George, L. (1996). Social Factors and Illness. Dans R.H. Binstock \& L.George (Éds.). Handbook of Aging and the Social Sciences (4e éd.) (pp. 229-248). San Diego: AcademicPress Inc. 